\section{A Survey of Fruit Firmness in Highbush Blueberry and Species- introgressed Blueberry Cultivars}

\author{
Mark K. Ehlenfeldt ${ }^{1}$ and Robert B. Martin Jr. ${ }^{2}$ \\ U.S. Department of Agriculture, Agricultural Research Service, Philip E. \\ Marucci Center for Blueberry and Cranberry Research and Extension at \\ Rutgers University, 125A Lake Oswego Road, Chatsworth, NJ 08019
}

Additional index words. Vaccinium angustifolium, V. ashei, V. corymbosum, V. darrowi, V. tenellum, fruit quality, rabbiteye, ripening

\begin{abstract}
Eighty-seven highbush blueberry and species-introgressed blueberry cultivars were evaluated for fruit firmness in the 1998-2000 growing seasons with a FirmTech 1 automated firmness tester. Significant differences were observed among cultivars. An average firmness of $136.1 \mathrm{~g} \cdot \mathrm{mm}^{-1}$ of deflection $\left(\mathrm{g} \cdot \mathrm{mm}^{-1} \mathrm{dfl}\right)$ was observed across all studied cultivars, and a range of $80.4 \mathrm{~g} \cdot \mathrm{mm}^{-1} \mathrm{dfl}$ ('Herbert') to $189.0 \mathrm{~g} \cdot \mathrm{mm}^{-1} \mathrm{dfl}$ ('Pearl River'). Species ancestry was not consistently related to firmness; however, cultivars with higher firmness values often possessed a higher percentage of Vaccinium darrowi Camp and $V$. ashei Reade ancestry. Conversely, cultivars with softer than average fruit often possessed a higher percentage of lowbush ( $V$. angustifolium Ait.) ancestry. This information may help to identify sources of breeding material for increased firmness in highbush blueberry hybrids.
\end{abstract}

In blueberry, softening that occurs at ripening is associated with the enzymatic digestion of cell wall components such as pectin, cellulose and hemicellulose (Proctor and Miesle, 1991). Total water soluble pectin, which comprises much of the middle lamella, was shown by Proctor and Peng (1989) to decrease steadily as blueberries ripen from green to blue. This degradation of the cell wall and middle lamella is directly responsible for the loss of firmness in ripening fruits (Eskin, 1979). Ripening is also accompanied by changes in coloration, decreases in acidity and increases in sugars (Kushman and Ballinger, 1968). Once initial ripeness has been achieved, a further process of "overripe softening" occurs which is accompanied by further decreases in acidity and increases in sugars. Both the innate firmness and post-ripeness softening are critical factors in the final quality of fresh blueberries.

Fruit firmness is an important economic trait in blueberry. Currently, labor accounts for $\approx 50 \%$ of the production cost of handharvested fresh-market fruit (Paul Macrie, N.J. blueberry grower, personal communication). Reductions in available farm labor, as well as increases in the minimum wage, have resulted in increased need for mechanical harvesting (Ballington et al., 1990). Firm fruit withstands both mechanical harvesting and subsequent shipping better than soft fruit (Ballington et al., 1984). Fruit of firmer cultivars can also be left on the bush longer (hanging potential) than fruit of soft cultivars, allowing more flexibility in timing of harvests. In addition to these production concerns, consumers per-

Received for publication 8 Nov. 2000. Accepted for publication 16 May 2001.

${ }^{1}$ Research Geneticist (Plants).

${ }^{2}$ Research Associate. ceive firm blueberries to be of higher quality. This perception (or the lack of it) can affect blueberry marketability and consumer demand. cultivated and species material. Ballington et al. (1984) evaluated 11 species, including $V$. corymbosum and $V$. ashei, and found that $V$. darrowi, V. tenellum Ait., V. myrtsinites Lam., and particularly $V$. stamineum L., are among the more firm-fruited species. In a comparison of $13 \mathrm{~V}$. ashei and $15 \mathrm{~V}$. corymbosum cultivars, $V$. ashei types were shown to be significantly firmer (Makus and Morris, 1987). Conversely, Vaccinium angustifolium has been shown to be less firm than $V$. corymbosum (Ballington et al., 1984).

Firmness is an exceedingly important characteristic when evaluating for cultivar release. Parental phenotype in blueberry often determines progeny firmness characteristics (Edwards etal., 1974). Vacciniumangustifolium, for instance, was shown by Finn and Luby (1992) to produce soft-fruited progeny. Several cultivars released in the past two decades have displayed greater firmness than earlier cultivars. 'Duke' (Draper et al., 1987), 'Sierra' [U.S. Dept. of Agriculture (USDA), 1988], 'Nelson' (USDA, 1988), and 'Reveille' (Ballington et al., 1990) all displayed superior firmness. Cultivars also differ in their ability to retain firmness after initial ripening. In recent years, automated firmness testers have allowed for objective measurement of fruit firmness (Mitcham et al., 1998).

The USDA-Agricultural Research Service (ARS) blueberry breeding program at the Philip E. Marucci Center for Blueberry and Cranberry Research and Extension at Rutgers Univ. has an extensive collection of highbush blueberry cultivars. We felt the first step in breeding for improved firmness was to assay the
Fruit firmness has been evaluated in both collection for firmness of ripe fruit prior to softening. This study was done to measure the firmness of a large number of blueberry cultivars and to examine firmness in relation to ancestry and release date.

\section{Materials and Methods}

Fruit samples of 87 blueberry cultivars were assayed across three growing seasons, 1998 to 2000, from demonstration plots planted in 1995 at the Philip E. Marucci Center for Blueberry and Cranberry Research and Extension at Rutgers Univ., Chatsworth, N.J. The bushes were grown in USDA plant hardiness zone 6, on soils that are mostly Atsion sand containing $3 \%$ to $15 \%$ organic matter. Cultural practices in the plots $(3 \mathrm{~cm}$ deep $\times$ $0.75 \mathrm{~m}$ wide) included clean cultivation, surface mulching with cranberry leaves, and the use of solid-set irrigation for irrigation and frost protection. In these plots, northernadapted highbush cultivars were represented by five plants per cultivar (among these were 'Legacy', 'Sierra', and 'Ozarkblue', all of which have some $V$. darrowi ancestry). All other cultivars, including North Carolinaadapted highbush, southern highbush $(V$. corymbosum introgressed with $V$. darrowi), half-highs ( $V$. angustifolium $\times V$. corymbosum hybrids), processing types ( $V$. ashei $\times V$. constablaei Gray), and rabbiteye hybrids ( $V$. ashei $\times V$. corymbosum), were represented by two plants (except as noted in Table 1). The cultivars were evaluated weekly for overall ripeness, and ripe fruit was sampled a single time when the crop on that cultivar was $15 \%$ to $25 \%$ ripe. Fruit was sampled equally from the available bushes, and care was taken to ensure that the fruit collected was undamaged and at peak quality. Thirty-five to forty berries were collected from each cultivar in 1998 and 60 70 berries were collected in 1999 and 2000 . After collection, the samples were refrigerated at $5{ }^{\circ} \mathrm{C}$ until the next day, then brought to $21{ }^{\circ} \mathrm{C}$ before being evaluated. A FirmTech 1 firmness tester (BioWorks, Stillwater, Okla.), which measured the force-deformation responses of compressed berries in terms of $\mathrm{g} \cdot \mathrm{mm}^{-1}$ of deflection $\left(\mathrm{g} \cdot \mathrm{mm}^{-1} \mathrm{dfl}\right)$, was used to assess firmness. The testing unit was set to a minimum deflection threshold of $15 \mathrm{~g}$ and a maximum compression force of $200 \mathrm{~g}$. In 1998, each sample size was 25 berries and in 1999 and 2000 sample size was 50. To minimize structural effects, berries were positioned so they would be compressed equatorially. The FirmTech 1 tester reported average firmness in each experimental sample as well as standard deviation, minimum and maximum values. Additionally, firmness values for individual berries were stored to spreadsheet files that were accessed for statistical analyses.

A square root transformation was applied to equalize the variances within the data before statistical analysis. Data were analyzed as a two-factor general linear mixed model using the Proc Mixed model of the Statistical Analysis System (SAS Institute, Cary, N.C.), with cultivars as a fixed factor, and years as a random factor. Means comparisons were per- 
formed with the Tukey-Kramer adjusted $P$ test $(P \leq 0.05)$.

\section{Results and Discussion}

For firmness, year was not a significant effect; however, there was a significant interaction of year $\times$ cultivar $(\mathrm{F}=8.18, P \leq 0.0001)$. Yearly averages for 1998 and 1999 were closely comparable at 141.7 and $140.4 \mathrm{~g} \cdot \mathrm{mm}^{-1} \mathrm{dfl}$, respectively, whereas the 2000 average was somewhat lower at $131.1 \mathrm{~g} \cdot \mathrm{mm}^{-1} \mathrm{dfl}$ (Table 1 ). The lower firmness in 2000 may be linked to weather differences, in that, among the 3 years, the 2000 growing season had average to above average rainfall, while both the 1998 and 1999 seasons had rainfall considerably below average. Drier conditions may have led to the formation of thicker cuticles on fruit. Cuticle and epidermal thickness may have played a role in measured fruit firmness. Shrinking and swelling of berries in response to turgor changes may also have caused the fruit to soften. The effect of weather on blueberry firmness is poorly understood; however, Ballinger et al. (1973) found both year and harvest date effects could be significant.

Significant differences were found for cultivar firmness $(\mathrm{F}=5.95, P \leq 0.0001)$. Average firmness values ranged from $80.4 \mathrm{~g} \cdot \mathrm{mm}^{-1} \mathrm{dfl}$ ('Herbert') to $189.0 \mathrm{~g} \cdot \mathrm{mm}^{-1} \mathrm{dfl}$ ('Pearl River') with an average of all cultivars $136.7 \mathrm{~g} \cdot \mathrm{mm}^{-1} \mathrm{dfl}$ (Table 1). Because of the very large number of pairwise comparisons made among cultivars, and the relatively conservative nature of the Tukey-Kramer test, 14 statistical groupings were resolved and considerable overlap existed between groups. The firmest group contained 'Pearl River', 'Misty' (186.7 g. $\mathrm{mm}^{-1}$ dfl), 'Bladen' (184.7 g.mm ${ }^{-1} \mathrm{dfl}$ ), 'Cooper' (174.5 g. $\left.\mathrm{mm}^{-1} \mathrm{dfl}\right)$, and 'Avonblue' (172.4 $\mathrm{g} \cdot \mathrm{mm}^{-1} \mathrm{dfl}$ ) as well as 44 other cultivars, and ranged as low as $129.0 \mathrm{~g} \cdot \mathrm{mm}^{-1} \mathrm{dfl}$ ('Ornablue'). The softest group contained
'Herbert' (80.4 g. $\left.\mathrm{mm}^{-1} \mathrm{dfl}\right)$, 'Atlantic' (98.8 $\mathrm{g} \cdot \mathrm{mm}^{-1} \mathrm{dfl}$ ), and 20 additional cultivars, and ranged as high as $129.0 \mathrm{~g} \cdot \mathrm{mm}^{-1} \mathrm{dfl}$ ('Ornablue'). Despite the lack of statistical separation among cultivars the casual observer can normally recognize firmness differences of $10-20 \mathrm{~g} \cdot \mathrm{mm}^{-1}$ dfl (personal observation). In our evaluations, firmness of $130-140 \mathrm{~g} \cdot \mathrm{mm}^{-1} \mathrm{dfl}$ is considered average, and typical of several widely grown cultivars such as 'Bluecrop', 'Elliott', and 'Croatan'. Firmness of $150-160 \mathrm{~g} \cdot \mathrm{mm}^{-1} \mathrm{dfl}$ is very good and typical of cultivars such as 'Duke', 'Burlington', 'Ozarkblue', 'Reka', 'Star', and 'Brigitta Blue'. Values above 160 are considered superior.

Differences in climate, soils, cultural practices, and other environmental factors may cause firmness of some cultivars to vary from region to region. For instance, studies conducted in North Carolina have shown that 'Reveille' is consistently firmer than 'Bladen', and 'Croatan' is, on average, much softer than

Table 1. Fruit firmness values of highbush blueberry (Vaccinium corymbosum) and species-introgressed blueberry cultivars, 1998-2000.

\begin{tabular}{|c|c|c|c|c|c|c|c|c|}
\hline \multirow[b]{2}{*}{ Cultivar } & \multirow[b]{2}{*}{ Type $^{z}$} & \multirow{2}{*}{$\begin{array}{c}\text { Year of } \\
\text { release }\end{array}$} & \multicolumn{6}{|c|}{ Firmness $\left(\mathrm{g} \cdot \mathrm{mm}^{-1}\right.$ of deflection) } \\
\hline & & & Avg & 1998 & 1999 & 2000 & Min. & Max. \\
\hline Herbert & HB & 1952 & $80.4 \mathrm{a}^{\mathrm{y}}$ & 79.1 & 91.5 & 73.4 & 53.4 & 118.0 \\
\hline Atlantic & $\mathrm{HB}$ & 1939 & $98.8 \mathrm{ab}$ & 89.2 & 109.9 & 100.1 & 71.0 & 141.1 \\
\hline Cabot & HB & 1920 & $103.1 \mathrm{a}-\mathrm{d}$ & 112.1 & --- & 93.7 & 67.9 & 146.7 \\
\hline Concord & $\mathrm{HB}$ & 1928 & $103.7 \mathrm{a}-\mathrm{c}$ & 97.6 & 109.8 & 106.1 & 71.9 & 145.4 \\
\hline Murphy & HB & 1950 & $105.0 \mathrm{a}-\mathrm{d}$ & 96.2 & --- & 114.1 & 81.6 & 139.4 \\
\hline Dixi & $\mathrm{HB}$ & 1936 & $107.8 \mathrm{a}-\mathrm{d}$ & 104.6 & 109.9 & 111.5 & 75.5 & 163.4 \\
\hline Wareham & HB & 1936 & $108.3 \mathrm{a}-\mathrm{d}$ & 107.7 & 121.6 & 98.4 & 67.9 & 150.7 \\
\hline Olympia & $\mathrm{HB}$ & 1933 & $112.3 \mathrm{a}-\mathrm{e}$ & 126.8 & 100.0 & 112.9 & 81.3 & 150.1 \\
\hline Northland & HB & 1967 & $113.3 \mathrm{a}-\mathrm{f}$ & 105.6 & 121.2 & 114.7 & 89.8 & 147.5 \\
\hline Hardyblue & $\mathrm{HB}$ & --- & $113.8 \mathrm{a}-\mathrm{f}$ & 124.7 & 110.4 & 108.5 & 79.6 & 153.7 \\
\hline Wolcott & $\mathrm{HB}$ & 1950 & $114.9 \mathrm{a}-\mathrm{f}$ & 107.3 & 129.8 & 110.5 & 83.5 & 158.4 \\
\hline St. Cloud & $\mathrm{HH}$ & 1990 & $115.3 \mathrm{a}-\mathrm{i}$ & --- & 115.0 & 113.2 & 88.7 & 148.6 \\
\hline Ivanhoe & HB & 1951 & $115.8 \mathrm{a}-\mathrm{f}$ & 131.3 & 106.1 & 114.2 & 79.6 & 176.5 \\
\hline Friendship & $\mathrm{HH}$ & 1992 & $118.5 \mathrm{a}-\mathrm{n}$ & --- & 122.1 & $107.1^{x}$ & 79.7 & 135.1 \\
\hline Pioneer & HB & 1920 & $119.4 \mathrm{a}-\mathrm{g}$ & 107.7 & 134.8 & 118.5 & 87.0 & 168.3 \\
\hline Georgiagem & SHB & 1987 & $119.9 \mathrm{a}-\mathrm{h}$ & 127.1 & 128.8 & 106.4 & 89.8 & 159.2 \\
\hline Heerma & $\mathrm{HB}$ & 1983 & $120.6 \mathrm{a}-\mathrm{k}$ & --- & 129.9 & 111.8 & 72.7 & 183.3 \\
\hline Patriot & $\mathrm{HB}$ & 1976 & $121.5 \mathrm{a}-\mathrm{i}$ & 121.3 & 126.0 & 118.6 & 95.5 & 165.0 \\
\hline Bluejay & HB & 1978 & $121.5 \mathrm{a}-\mathrm{i}$ & 123.8 & 122.8 & 118.7 & 88.5 & 164.1 \\
\hline Sampson & SHB & 1998 & $122.2 \mathrm{~b}-\mathrm{i}$ & 138.0 & 107.0 & 125.5 & 86.3 & 199.2 \\
\hline Jersey & HB & 1928 & $123.8 \mathrm{~b}-\mathrm{j}$ & 130.0 & 112.8 & 131.0 & 86.3 & 164.2 \\
\hline Weymouth & HB & 1936 & $124.1 \mathrm{~b}-\mathrm{j}$ & 124.8 & 117.2 & 133.1 & 76.6 & 170.9 \\
\hline Stanley & $\mathrm{HB}$ & 1930 & $124.1 \mathrm{~b}-\mathrm{j}$ & 123.6 & 121.3 & 129.2 & 91.5 & 167.5 \\
\hline Elizabeth & HB & 1966 & $125.2 \mathrm{~b}-1$ & 110.3 & 137.6 & 116.8 & 87.6 & 157.9 \\
\hline June & HB & 1930 & $125.6 \mathrm{~b}-\mathrm{k}$ & 121.1 & 135.9 & 121.2 & 85.8 & 159.0 \\
\hline Blueray & HB & 1955 & $125.9 \mathrm{~b}-\mathrm{k}$ & 110.3 & 135.9 & 135.4 & 83.4 & 193.8 \\
\hline Bluetta & $\mathrm{HB}$ & 1967 & 127.2 b-k & 143.3 & 119.8 & 122.3 & 76.2 & 168.3 \\
\hline Harding & HB & 1912 & $127.5 \mathrm{~b}-1$ & --- & 126.5 & 126.6 & 86.9 & 176.5 \\
\hline Little Giant & PRO & 1995 & $127.8 \mathrm{~b}-1$ & 111.8 & 127.6 & 146.7 & 95.1 & 168.4 \\
\hline Sunrise & HB & 1988 & 128.4 b-1 & 128.4 & 137.7 & 121.6 & 93.4 & 182.8 \\
\hline Lateblue & HB & 1967 & 128.6 b-1 & 130.4 & 156.3 & 103.6 & 96.1 & 179.9 \\
\hline Puru & HB & 1989 & 128.7 b-1 & 146.6 & 113.9 & 128.2 & 92.5 & 163.7 \\
\hline Ornablue & ORN & 1969 & 129.0 a-n & --- & 141.5 & 117.2 & 107.5 & 159.5 \\
\hline Blue Ridge & SHB & 1987 & 129.4 b-n & 131.0 & --- & 126.1 & 87.2 & 172.3 \\
\hline Pender & $\mathrm{HB}$ & 1998 & 129.5 b-1 & 130.4 & 126.5 & 134.0 & 90.4 & 190.3 \\
\hline Angola & $\mathrm{HB}$ & 1951 & $130.0 \mathrm{~b}-1$ & 132.9 & 154.1 & 107.9 & 89.9 & 170.6 \\
\hline Ama & $\mathrm{HB}$ & 1983 & $130.2 \mathrm{~b}-\mathrm{n}$ & --- & 125.1 & 131.0 & 65.3 & 174.0 \\
\hline Berkeley & HB & 1949 & $130.3 \mathrm{~b}-1$ & 111.5 & 152.6 & 129.8 & 88.8 & 199.4 \\
\hline Morrow & $\mathrm{HB}$ & 1964 & $131.0 \mathrm{~b}-1$ & 147.1 & 127.8 & 120.8 & 89.6 & 171.9 \\
\hline Darrow & HB & 1965 & $131.1 \mathrm{~b}-1$ & 138.9 & 139.4 & 117.7 & 93.5 & 182.2 \\
\hline Cape Fear & SHB & 1987 & $131.5 \mathrm{~b}-\mathrm{n}$ & 141.3 & --- & 120.9 & 65.7 & 174.3 \\
\hline Bluechip & $\mathrm{HB}$ & 1979 & $132.0 \mathrm{~b}-1$ & 133.5 & 143.4 & 123.3 & 57.8 & 193.6 \\
\hline Katherine & $\mathrm{HB}$ & 1920 & $132.1 \mathrm{~b}-\mathrm{n}$ & --- & 160.3 & 103.7 & 97.1 & 174.1 \\
\hline Spartan & HB & 1977 & 132.4 b-1 & 148.0 & 127.9 & 124.8 & 87.2 & 190.4 \\
\hline Bluecrop & HB & 1952 & $133.3 \mathrm{~b}-\mathrm{m}$ & 155.1 & 139.1 & 109.6 & 96.6 & 172.1 \\
\hline Croatan & HB & 1954 & $133.4 \mathrm{~b}-\mathrm{m}$ & 134.5 & 142.2 & 126.9 & 91.8 & 168.6 \\
\hline \multirow{2}{*}{ Nelson } & $\mathrm{HB}$ & 1988 & $133.6 \mathrm{~b}-\mathrm{m}$ & 141.8 & 146.0 & 114.4 & 95.2 & 185.8 \\
\hline & & & & & & & \multicolumn{2}{|c|}{ Continued on next page } \\
\hline
\end{tabular}


in our determinations (C.M. Mainland, personal communication). Similar differences can be found in a study by Perkins-Veazie et al. (1995). It should be noted, however, that we sought to determine "innate" firmness (i.e., after color development, but prior to any significant subsequent softening), and some differences between our determinations and those of others may represent sampling technique or degree of post-ripeness softening.

The regression of cultivar fruit firmness on release date was highly significant $(P \leq 0.001)$ with $r^{2}=0.20$. This $r^{2}$ value is quite low; however, the large number of cultivars sampled allowed significance to be discerned. The relationship between firmness and release date is represented by the equation:

$$
\text { Firmness }_{\mathrm{g} / \mathrm{mm} \mathrm{dfl}}=0.36 \text { year }-578.05 \text {. }
$$

Thus, the average gain per decade was only 3.6 $\mathrm{g} \cdot \mathrm{mm}^{-1} \mathrm{dfl}$. Many of the least-firm cultivars are pure $V$. cormybosum and the seven softest cultivars were among those released before 1953. That the newer cultivars overall tend to be firmer, may be attributable, at least partially, to the diversification of highbush germplasm through the incorporation of firmer species material into $V$. corymbosum. However, it may also be due to repeated cycles of selection for firmness. With the advent of mechanical harvesting and the now worldwide distribution of blueberries, breeders have placed continued emphasis on producing firmfruited cultivars.

All of the cultivars we labeled as southern highbush have some $V$. darrowi ancestry in their pedigree, and some possess additional germplasm such as $V$. ashei. This ancestry is a common factor in some of the most firm cultivars. In particular, 'Misty', 'Bladen', and 'Reveille', which were among the firmestfruited cultivars, all contain $V$. ashei and a trace of $V$. tennellum in their background, as well as $V$. darrowi. The southern highbush cultivars with the lowest firmness values were 'Cape Fear' (131.5 g. mm ${ }^{-1}$ dfl), 'Blue Ridge' (129.4 g. $\left.\mathrm{mm}^{-1} \mathrm{dfl}\right)$, 'Sampson' (122.2 g. $\mathrm{mm}^{-1}$ $\mathrm{dfl}$ ), and 'Georgiagem' (119.9 $\left.\mathrm{g} \cdot \mathrm{mm}^{-1} \mathrm{dfl}\right)$.
'Cape Fear', 'Blue Ridge', and 'Sampson' possess $V$. angustifolium in their ancestry $(15.7 \%, 15.6 \%$, and $11 \%$, respectively) and 'Georgiagem' has 'Ashworth' as a great-grandparent (Ehlenfeldt, 1994). Darrow et al. (1960) reported that 'Ashworth' produced soft fruit and they believed 'Ashworth' may contain significant lowbush germplasm.

Other cultivars that possess significant amounts of $V$. angustifolium ancestry, specifically the "half-highs" $(\approx 15 \%$ to $50 \% \mathrm{~V}$. angustifolium), seem to show a propensity for producing softer fruit. Of the three half-highs evaluated, two had values in the $110-120 \mathrm{~g} \cdot \mathrm{mm}^{-}$ ${ }^{1}$ dfl range. 'Polaris' $\left(151.1 \mathrm{~g} \cdot \mathrm{mm}^{-1} \mathrm{dfl}\right)$ was a notable exception, however, being nearly as firm as 'Duke' (154.4 g. $\left.\mathrm{mm}^{-1} \mathrm{dfl}\right)$. Even some cultivars with less lowbush ancestry than the half-highs seem to produce less-firm berries. 'Bluetta', 'June', and 'Northland' all possess $25 \% \mathrm{~V}$. angustifolium ancestry, (Hancock, et al., 1982) and exhibited lower than average firmness values (Table 1).

Fruit firmness of a number of rabbiteye

Table 1. Continued.

\begin{tabular}{|c|c|c|c|c|c|c|c|c|}
\hline \multirow[b]{2}{*}{ Cultivar } & \multirow[b]{2}{*}{ Type $^{2}$} & \multirow{2}{*}{$\begin{array}{c}\text { Year of } \\
\text { release }\end{array}$} & \multicolumn{6}{|c|}{ Firmness $\left(\mathrm{g} \cdot \mathrm{mm}^{-1}\right.$ of deflection $)$} \\
\hline & & & Avg & 1998 & 1999 & 2000 & Min. & Max. \\
\hline Toro & $\mathrm{HB}$ & 1987 & $133.7 \mathrm{~b}-\mathrm{m}$ & 136.5 & 137.5 & 129.4 & 100.8 & 186.2 \\
\hline Elliott & HB & 1973 & $135.4 \mathrm{~b}-\mathrm{n}$ & $136.2^{x}$ & 149.9 & 120.6 & 99.0 & 175.4 \\
\hline Chanticleer & $\mathrm{HB}$ & 1997 & $135.5 \mathrm{~b}-\mathrm{n}$ & 139.6 & 130.9 & 140.3 & 92.6 & 180.6 \\
\hline Bonus & $\mathrm{HB}$ & --- & $135.8 \mathrm{~b}-\mathrm{n}$ & 127.7 & $132.8^{x}$ & 146.0 & 77.0 & 201.3 \\
\hline Rubel & $\mathrm{HB}$ & 1912 & $136.3 \mathrm{~b}-\mathrm{n}$ & 117.7 & 165.8 & 129.3 & 104.1 & 185.2 \\
\hline Rancocas & $\mathrm{HB}$ & 1926 & $136.3 \mathrm{~b}-\mathrm{n}$ & 131.3 & 142.1 & 137.9 & 75.8 & 176.6 \\
\hline Collins & $\mathrm{HB}$ & 1959 & $138.1 \mathrm{~b}-\mathrm{n}$ & 157.9 & 130.6 & 129.6 & 89.1 & 200.0 \\
\hline Jubilee & SHB & 1994 & $138.1 \mathrm{~b}-\mathrm{n}$ & 162.3 & 118.3 & 137.3 & 97.0 & 186.4 \\
\hline Bluegold & $\mathrm{HB}$ & 1989 & $138.6 \mathrm{~b}-\mathrm{n}$ & $147.2^{x}$ & 143.5 & 130.4 & 95.7 & 199.4 \\
\hline Pemberton & $\mathrm{HB}$ & 1939 & $140.1 \mathrm{~b}-\mathrm{n}$ & 141.9 & 154.6 & 126.1 & 99.6 & 173.9 \\
\hline Bounty & HB & 1987 & $142.1 \mathrm{~b}-\mathrm{n}$ & 163.3 & --- & 121.7 & 102.5 & 189.6 \\
\hline Sharpblue & SHB & 1976 & $143.3 \mathrm{~b}-\mathrm{n}$ & 152.0 & 146.3 & 133.6 & 107.0 & 191.9 \\
\hline Earliblue & HB & 1952 & $143.4 \mathrm{~b}-\mathrm{n}$ & 173.9 & 130.4 & 124.3 & 96.4 & 205.0 \\
\hline Magnolia & SHB & 1994 & $146.1 \mathrm{c}-\mathrm{n}$ & 114.2 & 163.8 & 165.3 & 98.7 & 197.9 \\
\hline Legacy & $\mathrm{HB}$ & 1994 & $146.6 \mathrm{c}-\mathrm{n}$ & 149.2 & 145.5 & 132.2 & 108.2 & 183.8 \\
\hline Nui & $\mathrm{HB}$ & 1989 & $148.3 \mathrm{c}-\mathrm{n}$ & 156.6 & 150.4 & 145.6 & 94.6 & 194.2 \\
\hline O'Neal & SHB & 1987 & $148.6 \mathrm{c}-\mathrm{n}$ & 143.7 & 155.4 & 148.8 & 116.0 & 191.4 \\
\hline Polaris & $\mathrm{HH}$ & 1996 & $151.1 \mathrm{c}-\mathrm{n}$ & --- & 146.5 & 154.5 & 99.8 & 202.7 \\
\hline Sierra & $\mathrm{HB}$ & 1988 & $151.2 \mathrm{c}-\mathrm{n}$ & 173.5 & $148.9^{x}$ & 130.7 & 89.4 & 215.1 \\
\hline Meader & $\mathrm{HB}$ & 1971 & $151.9 \mathrm{~d}-\mathrm{n}$ & 164.5 & 147.2 & 146.8 & 109.8 & 210.5 \\
\hline Ozarkblue & SHB & 1996 & $153.2 \mathrm{~d}-\mathrm{n}$ & 147.4 & 169.9 & 144.3 & 119.3 & 214.7 \\
\hline Gulfcoast & SHB & 1987 & $153.5 \mathrm{~d}-\mathrm{n}$ & 160.3 & 148.7 & 155.1 & 91.9 & 217.0 \\
\hline Burlington & $\mathrm{HB}$ & 1939 & $153.5 \mathrm{~d}-\mathrm{n}$ & 182.8 & 152.8 & 131.0 & 103.2 & 215.0 \\
\hline Reka & HB & 1989 & $153.8 \mathrm{~d}-\mathrm{n}$ & 154.6 & 145.1 & 163.7 & 116.2 & 210.1 \\
\hline Summit & SHB & 1998 & $154.0 \mathrm{c}-\mathrm{n}$ & --- & 170.4 & 136.3 & 111.7 & 182.0 \\
\hline Chandler & $\mathrm{HB}$ & 1994 & $154.0 \mathrm{~d}-\mathrm{n}$ & 170.2 & 152.2 & 144.7 & 109.2 & 236.3 \\
\hline Coville & $\mathrm{HB}$ & 1949 & $154.2 \mathrm{~d}-\mathrm{n}$ & 148.2 & 175.8 & 142.3 & 100.9 & 221.7 \\
\hline Duke & $\mathrm{HB}$ & 1987 & $154.4 \mathrm{~d}-\mathrm{n}$ & 173.1 & 152.6 & 140.5 & 106.3 & 199.3 \\
\hline Star & SHB & 1996 & $155.9 \mathrm{~d}-\mathrm{n}$ & $181.3^{x}$ & 145.3 & 158.5 & 116.7 & 217.9 \\
\hline Scammel & $\mathrm{HB}$ & 1931 & $156.4 \mathrm{c}-\mathrm{n}$ & --- & 160.6 & 150.1 & 121.9 & 194.2 \\
\hline Brigitta Blue & $\mathrm{HB}$ & 1977 & $159.7 \mathrm{e}-\mathrm{n}$ & 188.0 & 150.2 & 145.9 & 99.3 & 230.9 \\
\hline Bluehaven & $\mathrm{HB}$ & 1967 & $161.4 \mathrm{f}-\mathrm{n}$ & 173.5 & 161.8 & 151.7 & 112.3 & 223.8 \\
\hline Harrison & $\mathrm{HB}$ & 1974 & $164.9 \mathrm{~g}-\mathrm{n}$ & 156.7 & 184.0 & 158.7 & 110.6 & 244.5 \\
\hline Marimba & SHB & 1992 & $169.0 \mathrm{~h}-\mathrm{n}$ & 175.6 & 164.8 & 169.1 & 116.6 & 223.2 \\
\hline Reveille & SHB & 1990 & $169.3 \mathrm{i}-\mathrm{n}$ & 185.9 & 174.7 & 150.5 & 124.6 & 220.5 \\
\hline Avonblue & SHB & 1977 & $172.2 \mathrm{j}-\mathrm{n}$ & 195.0 & 161.0 & 163.8 & 113.3 & 240.4 \\
\hline Cooper & SHB & 1987 & $174.5 \mathrm{k}-\mathrm{n}$ & 184.5 & 184.2 & 157.6 & 132.6 & 223.1 \\
\hline Bladen & SHB & 1990 & $184.71-\mathrm{n}$ & 193.9 & 184.0 & --- & 137.7 & 243.7 \\
\hline Misty & SHB & 1989 & $186.7 \mathrm{n}$ & 188.8 & 208.3 & 162.7 & 145.3 & 255.9 \\
\hline Pearl River & SHB/RE & 1994 & $189.0 \mathrm{mn}$ & 225.0 & 196.7 & 176.5 & 125.6 & 292. \\
\hline Average $^{w}$ & & & 136.7 & 141.7 & 140.4 & 131.1 & & \\
\hline
\end{tabular}

${ }^{2} \mathrm{HB}=$ highbush, $\mathrm{SHB}=$ southern highbush, $\mathrm{LB}=$ lowbush, $\mathrm{HH}=$ half-high, $\mathrm{ORN}=$ ornamental, $\mathrm{PRO}=$ processing type, $\mathrm{RE}=$ rabbiteye. ${ }^{\mathrm{y}}$ Means comparisons were performed using the Tukey-Kramer adjusted $P$ test $(P \leq 0.05)$.

${ }^{x}$ Entry for which multi-fruit datafile was lost. Statistical analysis used the single yearly average value for this entry.

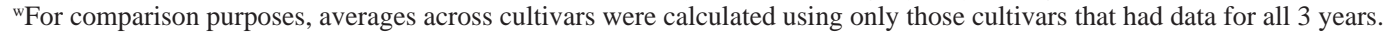


cultivars was evaluated during this study. These fruit, however, were collected from potted plants located in coldframes under daily sprinkler irrigation. Due to the differences in growing conditions, the values for $V$. ashe $i$ were not included in Table 1. However, the firmness of 7-13 rabbiteye cultivars evaluated across 3 years averaged $170.6 \mathrm{~g} \cdot \mathrm{mm}^{-1} \mathrm{dfl}$; considerably higher than the average of southern highbush cultivars $\left(151.6 \mathrm{~g} \cdot \mathrm{mm}^{-1} \mathrm{dfl}\right.$, excluding 'Pearl River'). 'Pearl River', which is 50\% V. ashei, was the firmest of the cultivars tested at $189.0 \mathrm{~g} \cdot \mathrm{mm}^{-1} \mathrm{dfl}$. These trends suggest that overall $V$. ashe $i$ is firmer than those cultivars derived from $V$. corymbosum and $V$. darrowi.

Introducing species germplasm into $V$. corymbosum and producing a commercially viable cultivar, although difficult and timeconsuming, seems to offer breeders a method of creating offspring with significantly improved firmness. However, there are also cultivars, such as 'Duke' that are firm-fruited, retain their firmness, and seem to transmit their firmness to progeny. In the USDA program, crosses have been made which attempt to incorporate the firmness present in $V$. ashei germplasm, 'Duke', and other sources.

Firmness has the potential to affect harvest loss, storability and consumer acceptance. This study measured innate firmness; studies also need to be made to assess the abilities of different cultivars to maintain firmness after initial ripening has occurred. Ehlenfeldt (1998) noted that several cultivars, including 'Duke', G-695 (released in 2000 as 'Cara's Choice'), and G-805 (released in 2000 as 'Hannah's Choice'), appear to maintain their firmness even when left on the bush. 'Reveille', in our recent observations, should also be added to the list of cultivars with excellent on-bush firmness retention. Postharvest softening is also a factor in need of further study. Although data concerning postharvest softening of blueberry in cold storage shows variable cultivar responses, Forney et al. (1998) showed that 'Burlington' gets firmer in storage. Other factors being equal, it seems likely that firmfruited, nonsoftening cultivars will store longer than the softer cultivars, and that any improvements in firmness will be beneficial to the industry.

\section{Literature Cited}

Ballinger, W.E., L.J. Kushman, and D.D. Hamann. 1973. Factors affecting the firmness of highbush blueberries. J. Amer. Soc. Hort. Sci. 98:583587.

Ballington, J.R., W.E. Ballinger, W.H. Swallow, G.J. Galletta, and L.J. Kushman. 1984. Fruit quality characterization of 11 Vaccinium species. J. Amer. Soc. Hort. Sci. 109:684-689.

Ballington, J.R., S.D. Rooks, and C.M. Mainland. 1990. 'Reveille,' a new southern highbush blueberry cultivar for mechanical harvesting for both fresh and processing market outlets. Proc. 24th Ann. Open House, Southern Blueberry Council: 7-16.

Darrow, G.M., L. Whitton, and D.H. Scott. 1960. The 'Ashworth' blueberry as a parent in breeding for hardiness and earliness. Fruit Var. and Hort. Digest 14:43-46.

Draper, A., G. Galletta, G. Jelenkovic, and N. Vorsa. 1987. 'Duke' highbush blueberry. HortScience 22:320.

Edwards, T.W., W.B. Sherman, and R.H. Sharpe. 1974. Evaluation and inheritance of fruit color, size, scar, firmness and plant vigor in blueberry. HortScience 9:20-22.

Ehlenfeldt, M.K. 1998. Breeding for fruit quality
parameters-How far have we come; how far can we go? Proc. 8th N. Amer. Blueberry Res. and Ext. Workers Conf.:24-29.

Ehlenfeldt, M.K. 1994. The genetic composition and tetrasomic inbreeding coefficients of highbush blueberry cultivars. HortScience 29:1342-1345.

Eskin, N.A.M. 1979. The plant cell wall, p. 123-138. In: N.A.M. Eskin (ed.). Plant pigments, flavors and textures: Textural components of food. Academic, New York.

Finn, C.E. and J.J. Luby. 1992. Inheritance of fruit quality traits in blueberry. J. Amer. Soc. Hort. Sci. 117:617-621

Forney, C.F. U.K.G.N. Kumudini, and M.A. Jordan. 1998. Effects of postharvest storage conditions on firmness of 'Burlington' blueberry fruit. Proc. 8th N. Am. Blueberry Res. and Ext. Workers Conf.:227-232

Hancock, J.F. and J.H. Sieker. 1982. Levels of inbreeding in highbush blueberry cultivars. HortScience 17:363-366.

Kushman, L.J. and W.E. Ballinger. 1968. Acid and sugar changes during ripening in Wolcott blueberries. Proc. Amer. Soc. Hort. Sci. 92:290-295.

Makus, D.J. and J.R. Morris. 1987. Highbush vs. rabbiteye blueberry: A comparison of fruit quality. Ark. Farm Res. May-June:5-6.

Mitcham, E.J., M. Clayton, and W.V. Biasi. 1998. Comparison of devices for measuring cherry fruit firmness. HortScience 33:723-727.

Perkins-Veazie, P., J.R. Clark, J.K. Collins, and J. Magee. 1995. Southern highbush blueberry clones differ in postharvest fruit quality. Fruit Var. J. 49:46-52.

Proctor, A. and T.J. Miesle. 1991. Polygalacturonase and pectinmethylesterase activities in developing highbush blueberries. HortScience 26:579-581.

Proctor, A. and L.C. Peng. 1989. Pectin transitions during blueberry fruit development and ripening. J. Food Sci. 54:385-387.

U.S. Department of Agriculture. 1988. Notice of release of 'Nelson' and 'Sierra' highbush blueberries. U.S. Dept. of Agr., Beltsville, Md. 